\title{
Effect of Ovary Lipid of Skipjack Tuna (Katsuwonus pelamis) on Anxious Behavior in Rats
}

\author{
Katumasa OOKAWA ${ }^{1)}$, Kazuo MOCHIZUKI ${ }^{1)}$, Eiji SHIDA ${ }^{1)}$, Toshihiro SUZUKI ${ }^{1)}$, Toshihiro SUZUKI ${ }^{1)}$, \\ Tomoko KITAGAWA ${ }^{2)}$, Toru MATUMOTO ${ }^{2)}$, Yoshinori HOKARI $^{2)}$ and Hidehiko YOKOGOSHI ${ }^{3)}$ \\ ${ }^{1)}$ Shizuoka Industrial Research Institute of Shizuoka Prefecture, 2078 Makigaya, Aoi, Shizuoka 421-1298, ${ }^{2)}$ Maruhachi Muramatsu, Inc. \\ 65-1 Jonokoshi, Yaizu, Shizuoka 425-0025 and ${ }^{3}$ University of Shizuoka, 52-1 Yada, Suruga, Shizuoka 422-8526, Japan
}

(Received 1 May 2006/Accepted 6 July 2006)

ABSTRACT. Ovary lipid of Skipjack tuna (OLS) (Katsuwonus pelamis) contains a high level of docosahexaenoic acid combined with phospholipids. In this study, we examined the effect of OLS in male Wistar rats given OLS mixed in feed ( $0.9 \%)$ for 42 days, using an animal model of anxiety, the elevated T-maze test. The avoidance latency at the 1st trial was significantly shorter in the OLS ingestion group than in the control group. Those at the 2 nd and 3rd trials showed a similar tendency. There was almost no difference in escape latency at the 1st trial between the two groups but the escape latencies at the 2 nd and 3rd trials tended to be longer in the OLS group. These results suggested that OLS inhibits anxious behavior in rats.

KEY WORDS: anxiety, elevated T-maze test, ovary lipid.

Ovaries removed from Skipjack tuna as a processing byproduct in Shizuoka Prefecture contain 6-7\% (w/w) of lipid. Docosahexaenoic acid (DHA) accounts for about 35\% of fatty acid in the ovary lipid of Skipjack tuna (OLS). Extracted oil and fat contains $34 \%$ (w/w) of phospholipids and the phospholipids are characterized by the composition of phosphatidylcholine: phosphatidylethanolamine: lysophosphatidylcholine of 50\%: 20\%: 20\% (w/w). Although it is unknown whether phospholipids in OLS are absorbed into the body without any change in the form, studies on DHA and DHA combined with phospholipids [2, 4, 14] suggest that OLS ingestion has an effect on brain function.

Humans have anxiety disorders such as generalized anxiety disorder, panic disorder, posttraumatic stress disorder, phobia, and obsessive-compulsive disorder. Animals also have anxiety-related problematic behaviors that mimic human anxiety disorders [8]. If OLS ingestion relieves anxiety in companion animals, it could be useful in the treatment of such problematic behaviors.

The elevated T-maze test is an excellent method for separately observing the anxiety/fear that animals subconsciously and instinctively have and that is caused by their memory $[3,5,9,13,14]$. We thought that if we could reveal which of the anxious/fear behaviors noted above receives a beneficial effect from OLS when OLS acts on anxious/fear behavior, we could demonstrate what kind of human and animal mental disease OLS could treat. We studied the effect of long-term OLS ingestion on behaviors in rats, using an animal anxiety model, the elevated T-maze test.

The ovary was taken out from Skipjack tuna (Katsuwonus pelamis), washed with water, boiled, freeze-dried, powdered, and subjected to extraction with ethanol. The resultant extract was filtered and concentrated to obtain ovary lipid of Skipjack tuna (OLS).

Six-week-old male Wistar rats (Japan SLC, Inc.) were used in experiments. They were raised in an animal room maintained at a temperature of $23 \pm 1{ }^{\circ} \mathrm{C}$, humidity of $55 \%$, light/dark cycle of $12 \mathrm{hr}$ (lighting 7:00-19:00, automatically controlled) in Facility II at the Animal Experiment Center of the University of Shizuoka (Yada, Suruga-ku, Shizuokashi). The rats were divided into the control group $(n=11)$ and OLS ingestion group $(n=11)$ and given test feeds for 42 days. In grouping them, the avoidance latency of each rat was measured once in advance, and the mean value of the control group and the OLS group were made similar (Control: $11.9 \pm 0.7 \mathrm{~s}$, OLS: $12.9 \pm 0.6 \mathrm{~s}, \mathrm{~F}$-test: $\mathrm{P}=0.76$, t-test: $\mathrm{P}=0.73$ ). The test feeds were prepared according to AIN93 and given to the animals ad libitum. The oil and fat contents of the test feeds are shown in Table 1. The contents were calculated to give the same energy percentages for $n-6$ and n-3 fatty acids and n-6/n-3 ratio. This study was conducted after approval by the Animal Experiment Center Steering Committee at the University of Shizuoka, in compliance with the Guidance for Animal Experiments in the University of Shizuoka.

The elevated T-maze was set at a height of $70 \mathrm{~cm}$ from the floor. The enclosed arm was $40 \mathrm{~cm}$ in wall height, $50 \mathrm{~cm}$ in length, and $9 \mathrm{~cm}$ in width. The two open arms with a ledge of $3 \mathrm{~mm}$ were $50 \mathrm{~cm}$ in length and $9 \mathrm{~cm}$ in width. The illumination intensity was 0.6 lux on the surfaces of the

Table 1. Lipid composition of tested diets

\begin{tabular}{|c|c|c|c|}
\hline Lipids & & Control & OLS \\
\hline Corn oil & $(\%)$ & - & 1.20 \\
\hline Rapeseed oil & $(\%)$ & 3.00 & - \\
\hline Sunflower oil & $(\%)$ & 2.00 & 2.90 \\
\hline Ovary lipids of skipjack tun & $(\%)$ & - & 0.90 \\
\hline n-3 fatty acid & \multirow{3}{*}{$\begin{array}{l}\text { (energy\%) } \\
(\text { energy\%) }\end{array}$} & 0.79 & 0.81 \\
\hline n-6 fatty acid & & 1.62 & 1.67 \\
\hline$n-6 / n-3$ & & 2.1 & 2.1 \\
\hline
\end{tabular}


enclosed and open arms. Prior to testing, a rat was transferred from the raising cage to a test cage and the test cage was left in the test room for $1 \mathrm{~min}$ for acclimation. The rat after acclimation was put on an end of the enclosed arm and the time to when the four limbs of the rat were out into the open arm area was measured as the avoidance latency. The observation time was $600 \mathrm{sec}$. When the rat moved or did not move to the open arm within $600 \mathrm{sec}$, it was transferred to the test cage. This test was repeated 3 times at intervals of $1 \mathrm{~min}$. After completing the measurement of avoidance latency, the rat was transferred to the test cage. At an interval of $1 \mathrm{~min}$, the rat was put on an end of the open arm area to measure as the escape latency the time to when the four limbs were into the enclosed arm. The observation time was 180 seconds. The escape latency was measured 3 times in the same way as for avoidance latency [13]. The rats were decapitated after the elevated T-maze test to obtain serum. Serum total cholesterol (Cho), triglyceride (TG), HDL-cholesterol (HDL), phospholipids (PL), and non-esterified fatty acid (NEFA) were measured using Cholesterol E-Test Wako, Triglyceride E-Test Wako, HDL-Cholesterol E-Test Wako, Phospholipid C-Test Wako, and NEFA C-Test Wako (purchased from Wako Pure Chemical Industries, Ltd.) as directed in the attached leaflets $[6,7,10]$.

Data were expressed as a mean \pm standard error (SE). Each item of data obtained on avoidance latency and escape latency was examined using the nested model ANOVA [16]. When there was a significant difference on the group or time factor, the difference between the control group and OLS group in each of the 1st, 2nd, and 3rd trials was examined using the one-sided Mann-Whitney U-test (5\% level). Serum chemistries were analyzed at a significant level of 5\% using the one-sided Mann-Whitney U-test.

As shown in Table 2, Cho, HDL and PL concentration was significantly lower in the OLS ingestion group than in
Table 2. Serum chemistries of rats

\begin{tabular}{lccc}
\hline & & $\begin{array}{c}\text { Control } \\
(\mathrm{n}=11)\end{array}$ & $\begin{array}{c}\text { OLS } \\
(\mathrm{n}=11)\end{array}$ \\
\hline Total cholesterol & $(\mathrm{mg} / \mathrm{d} l)$ & $78.4 \pm 2.6$ & $66.2 \pm 3.8^{*}$ \\
Triglyceride & $(\mathrm{mg} / \mathrm{d} l)$ & $84.4 \pm 7.1$ & $109.7 \pm 11.1$ \\
HDL-cholesterol & $(\mathrm{mg} / \mathrm{d} l)$ & $42.3 \pm 4.0$ & $32.3 \pm 1.5^{*}$ \\
Phospholipid & $(\mathrm{mg} / \mathrm{d} l)$ & $129.5 \pm 5.2$ & $110.3 \pm 6.2^{*}$ \\
Non esterified fatty acid $(\mathrm{mEq} / l)$ & $0.61 \pm 0.08$ & $0.70 \pm 0.05$
\end{tabular}

Data is expressed as mean \pm S.E. *: Significantly different from the control group at $\mathrm{P}<0.05$.

the control group. It is already known that OLS reduces the Cho and HDL of few-week-old rats, and we got the same results this time. As for PL, it was thought to be increased by OLS ingestion, but it was adversely reduced. We need to conduct further studies on this.

Figure 1 shows results for avoidance latency and escape latency. The nested model ANOVA shows that there is a difference both in avoidance latency and escape latency between the group at a $1 \%$ significant level, and there is a difference in behavior facing anxiety/fear between the control group and OLS group. The avoidance latency at the $1 \mathrm{st}$ trial was significantly shorter in the OLS ingestion group than in the control group. At the 2nd and 3rd trials, the mean value was lower in the OLS ingestion group than in the control group, although no significant difference was observed. We believe that the lower value shown in the OLS group than in the control group in the 1st trial indicates OLS has a greater effect on the anxiety/fear that animals have subconsciously and instinctively than their anxiety/fear caused by memory. In addition, there was a tendency that avoidance latency, which is considered to increase through repetition of trials, was lower in the 2 nd trial than in the 1 st trial both in the control group and OLS group. It is already confirmed
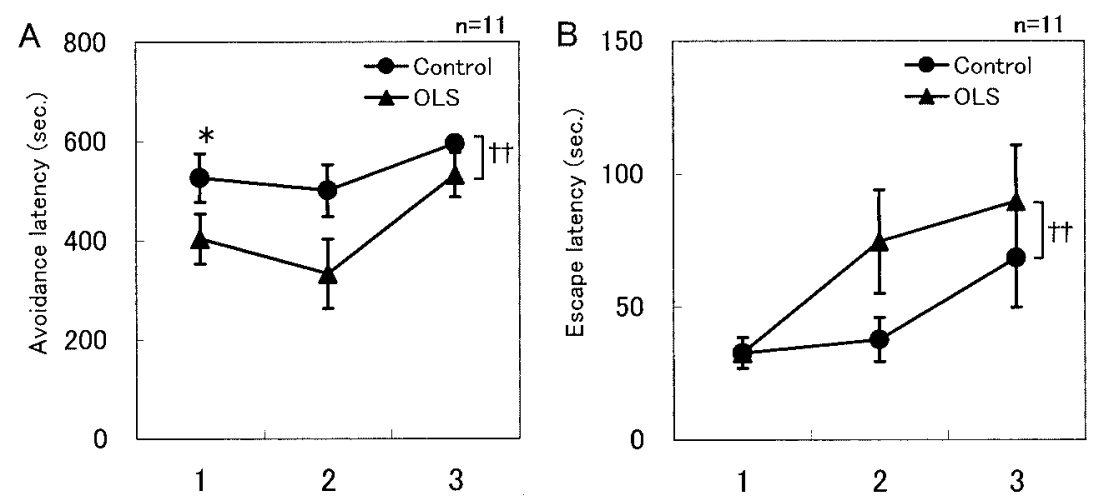

Fig. 1. Effect of ovary lipid of Skipjack tuna (OLS) on anxious behavior in rats. A: Avoidance latency, B: Escape latency. Data is expressed as mean \pm SE. Data were tested by the nested model ANOVA, and as for the data that was significantly different between the group and time, the difference between the control group and OLS group shown in each of the 1st, 2nd, and 3rd trials was examined by the one-sided Mann-Whitney U-test (5\% level). $\dagger \dagger$ : Significant difference between the control group and OLS group with the significant level by the nested model ANVOA set as $1 \%$. *: Significant difference between the control group and OLS group at the same time with the significant level set as $5 \%$. 
in similar experiments that repeated trials increase avoidance latency. Therefore we infer that $n-3$ fatty acid in the feed, a factor which is common to both the control group and OLS group and different from other reports, might have affected the 2 nd trial. The escape latency at the 1 st trial was short in the control and OLS ingestion groups without a difference. The escape latency at the 2 nd trial was longer than that at the 1st trial in the control and OLS ingestion groups. Although no significant difference was observed between the control and OLS ingestion groups, the value was higher in the latter group. The escape latency at the 3rd trial was even longer than that at the 2 nd trial in the control and OLS ingestion groups. In comparison between the two groups, the values had a similar tendency to those at the 2 nd trial.

These results demonstrate that OLS ingestion could suppress anxiety/fear. We would like to confirm the effect of OLS on anxiety/fear in the future by using other anxiety assessment methods such as an elevated plus-maze test.

There have never been detailed studies so far on the effect of n-3 fatty acid and n- 6 fatty acid in feed and their relationships using the elevated T-maze test. Certain data suggests that $n-3$ fatty acid has an anti-anxiety action, but what kind of anxiety it has efficacy for is not detailed [1, 11, 12]. As mentioned above, if n-3 fatty acid affected the repetition of trials on avoidance latency, avoidance latency assessed by the elevated T-maze test might be an effective method for studying the effect of n-3 fatty acid on anxiety in more detail.

\section{REFERENCES}

1. Belzung, C., Leguisquet, AM., Barreau, S., Delion-Vancassel, S., Chalon, S. and Durand, G. 1998. J. Nutr. 128: 1537-1542.

2. Carrie, I., Smirnova, M., Clement, M., Dejavel, D., Frances, H. and Bourre, J. M. 2002. Nutr. Neurosc. 5: 43-52.

3. Graeff, F. G., Viana, M. B. and Tomaz, C. 1993. Braz. J. Med. Biol. Res. 26: 67-70.

4. Hara, K. 1996. pp. 113-243. Biochemistry and Applications of Physiologically Active Lipids EPA and DHA (in Japanese).

5. Hernandes, A.S. and Almeida, S.S, 2003. Nutr. Neurosc. 6: 213-219.

6. Nagata, J., Kasai, M., Watanabe, S., Ikeda, I. and Saito, M. 2003. Biosci. Biotechnol. Biochem. 67: 1937-1943.

7. Nakasa, T., Ueda, S., Nakatsuka, M. and Okinaka, O. 2003. Nippon Shokuhin Kagaku Kogaku Kaishi 50: 463-467 (in Japanese).

8. Olivier, B. and Miczek, K.A. 2003. pp.105-121. Psychopharmacology of Animal Behavior Disorders.

9. Poltronieri, S.C., Zangrossi, H. Jr. and Viana, M.de B. 2003. Behav. Brain Res. 147: 185-192.

10. Shimada, Y., Yamakawa, A., Morita, T. and Sugiyama, K. 2003. Biosci. Biotechnol. Biochem. 67: 1258-1266.

11. Song, C., Leonard, B. E. and Horrobin, D. F. 2004. Stress 7: $43-54$.

12. Song, C., Li, X., Leonard, B. E. and Horrobin, D. F. 2003. J. Lipid Res. 44: 1984-1991.

13. Teixeira, R. C., Zangrossi, H.Jr. and Graeff, F.G. 2000. Pharmacol. Biochem. Behav. 65: 571-576.

14. Viana, M. B., Tomaz, C. and Graeff, F. G. 1994. Pharmacol. Biochem. Behav. 49: 549-554.

15. Yazawa, K. 1992. New Food Industry 34: 37-42 (in Japanese).

16. Yoshimura, I. and Oohashi, Y. 1992. pp. 63-93. Statistical Analysis for Toxicology Data (in Japanese). 